\title{
An audit of gastrostomy tube types and complication rates in home enteral tube-fed patients in Croydon Primary Care Trust (PCT)
}

\author{
M. Bhinda ${ }^{1}$, J. Nimmo ${ }^{2}$ and G. P. Hubbard ${ }^{2}$ \\ ${ }^{1}$ Department of Nutrition and Dietetics, Mayday University Hospital, London Rd, Thornton Heath CR7 7YE, UK \\ and ${ }^{2}$ Homeward Nurse Service and Medical Affairs Department, Nutricia, Whitehorse Business Park, Trowbridge, \\ Wilts BA14 OXQ, UK
}

The number of patients in the community with gastrostomy tube placement for home enteral tube feeding is increasing, with patients being fed via gastrostomy tube which can impact upon compliation and infection rates for longer periods of time ${ }^{(1)}$. There is some evidence that the type of gastrostomy tubes used can be associated with increased complications and infections ${ }^{(2,3)}$.

The aims of this audit were to examine the patient journey, identifying the number of complications related to the gastrostomy tube according to the type of tube being used and to assess the frequency of tube replacements in Croydon PCT. The audit was carried out on all patients registered on the Nutricia Homeward home enteral feeding service between July 2006 and July 2007 within Croydon PCT. Consent was received from each patient. Patients with a percutaneous endoscopic gastrostomy (PEG) or radiologically-inserted gastrostomy (RIG) were included, independent of which hospital had originally placed their PEG or RIG. The audit form was developed by both the community dietitian and the Nutricia homeward nurse and was completed from the patient's notes and on a routine review visit to the patient by the homeward nurse. The complications recorded included signs of infection or recurrent infection, granuloma or overgranulaton, gastric leakage and acid burn, inability to rotate or advance the tube, tube deterioration and recurrent replacements. The audit was completed for 100 patients, fifty-six were male and forty-four were female with an average age of 61 (range 19-95) years. The primary diagnosis of the audited patients included cerebrovascular accident, neurological diseases, cancer (head and neck), cerebral palsy and learning disabilities.

Tube type varied depending on which hospital had placed the gastrostomy. The majority of patients had either a Si-based (Vygon ${ }^{\circledR}$; Vygon (UK) Ltd, Cirencester, Glos., UK) $(40 \%)$ or polyurethane-based (Corflo ${ }^{\circledR}$; Merck Ltd, Whitehouse Station, NJ, USA) (40\%) tube type. Other types included Freka ${ }^{\circledR}$ (polyurethane; Fresenius Kabi, Bad Homburg, Germany) 7\%; Flocare ${ }^{\circledR}$ (Carbothane ${ }^{\circledR}$; Nutricia, Zoetermeer, The Netherlands) $2 \%$; or miscellaneous types $11 \%$. There were sixty-six incidences of tube-related complications in thirtyseven patients. Of the sixty-six tube-related complications, forty-eight $(73 \%)$ were in patients with the Si-based tube and sixteen $(24 \%)$ were in patients with the polyurethane tube $\left(\right.$ Corflo $\left.^{\circledR}\right)$. In all other tube types, only two $(3 \%)$ tube-related complications were observed. Patients with more than one complication had either the Si $\left(\right.$ Vygon ${ }^{\mathbb{R}}$ ) $(35 \%)$ or polyurethane (Corflo ${ }^{\mathbb{R}}$ ) $(10 \%)$ tube type. The most common tube-related complications included inability to rotate or advance the tube $(n 26 ; 39 \%)$ and tube deterioration $(n 10 ; 15 \%)$. A number of stoma site-related complications were observed, including redness and oozing ( $n$ 32) and granuloma or overgranulaton ( $n$ 9), but, in particular, five $(12.5 \%)$ patients experienced Candida-related infections and these were only recorded in patients with a Si-based tube. Forty-six tube replacements (approximately four per month) were carried out in the time period of the audit, of which $43 \%$ were of the Si-based tube.

The results from this audit highlight the different types of gastrostomy tube that are being used in the community and the complications and infections related to gastrostomy tubes. The results indicate that Si tube types appear to be associated with higher complication and infection rates and higher rates of tube replacement in clinical practice, all of which require more nursing time to manage. This finding indicates the need for the review of the types of gastrostomy tube used in clinical practice and for a comprehensive home enteral tube feeding service for patients, including regular reviews and stoma and tube care. A limitation to this audit may be that the placement methods in different hospitals varied, which may have affected the complication and replacement rates. As a result of this audit data it is intended to calculate the cost implications of these findings, with the aim of reviewing the types of tubes used, improvement in patient outcome and best use of financial resources.

1. British Artificial Nutrition Survey (2007) Artificial nutrition support in the UK 2000-2006. http://www.bapen.org.uk/pdfs/bans_reports/bans_report_ 07.pdf

2. Gottlieb K, Iber FL, Livak A et al. (1994) J Parenter Enteral Nutr 18, 264-267.

3. Iber FL, Livak A \& Patel M (1996) Dig Dis Sci 41, 226-231. 